\title{
Competencia de modelización en interpretación de gráficas funcionales
}

\section{Modeling competence in functional graph interpretation}

\author{
Horacio Solar \\ Departamento de Didáctica, Pontificia Universidad Católica de Chile. \\ hsolar@uc.cl \\ Jordi Deulofeu \\ Universitat Autònoma de Barcelona \\ jordi.deulofeu@uab.cat \\ Carmen Azcárate \\ Departament de Didàctica de les Matemàtiques i les Ciències Experimentals, Universitat Autònoma de Barcelona. \\ carmen.azcarate@uab.cat
}

RESUMEN • En este artículo presentamos una investigación en que se aplica el Modelo de Competencia Matemática (MCM) para estudiar la competencia de modelización, que se caracteriza por: tareas matemáticas, procesos y fases de la modelización. Las relaciones entre estos tres componentes determinan los niveles de complejidad cognitiva de una actividad matemática. Los procesos constituyen el componente más destacado y se desarrollan a lo largo de la etapa escolar, a diferencia de las tareas matemáticas, que se trabajan a corto plazo. El MCM se pone a prueba en un estudio de caso de una profesora que implementa una unidad didáctica de interpretación de gráficas funcionales.

PALABRAS CLAVE: competencias matemáticas; modelo de competencia matemática; modelización matemática; procesos matemáticos; interpretación de gráficas funcionales.

ABSTRACT - In this article we present a research where the Mathematics Competence Model (MCM) is applied to study the modeling competence, which is characterized by tasks, processes and modeling phases. The relationships between those three components determine the levels of cognitive complexity of a mathematical activity. The processes are the most outstanding components and they are developed during the whole school process, unlike the mathematical tasks, that are developed during shorter terms. The MCM is tested in a case study of a teacher who implemented a teaching unit for the interpretation of functional graphs.

KEYWORDS: mathematical competence; mathematical competence model; mathematical modeling; mathematical processes; functional graph interpretation.

Fecha de recepción: septiembre $2014 \bullet$ Aceptado: febrero 2015

Solar, H., Deulofeu, J., Azcárate, C. (2015) Competencia de modelización en interpreta-

ción de gráficas funcionales. Enseñanza de las Ciencias, 33.2, pp. 191-210 


\section{INTRODUCCIÓN}

El estudio de la modelización en el aula está cada vez más vinculado con la noción de competencia. En el 14 ICMI (Blum, Galbraith, Henn y Niss, 2007) las competencias en la modelización han sido interpretadas principalmente de dos maneras: como las acciones que se deben llevar a cabo en la fases de modelización (Kaiser, 2007) y como niveles de complejidad (Henning y Keune, 2007). En cambio no hay un consenso en el tratamiento de aspectos didácticos de la modelización, tales como: criterios para estudiar la comprensión de los estudiantes al trabajar con modelos, los ejemplos de actividades para que emerjan modelos matemáticos y el rol de profesor.

En nuestro estudio, la competencia de modelización tiene el propósito de, valga la redundancia, ser desarrolladora de competencias, e incorpora las dos visiones sobre competencias antes mencionadas. A su vez tiene un propósito de cumplir una función didáctica, ya que involucra aspectos en relación al estudiante, a la actividad matemática y al rol del profesor.

La modelización matemática la consideramos como un proceso articulador del currículum, y en otros trabajos (Espinoza, Barbé, Mitrovich, Solar, Rojas y Matus, 2008; Solar, Azcárate y Deulofeu, 2012) se ha justificado que el enfoque por competencias es un sustento para identificar procesos articuladores de aquel. El enfoque por competencias, además de promover un enfoque funcional de las matemáticas, caracteriza la modelización matemática como un proceso, y esta condición da pie a elaborar un modelo de competencia matemática que trate aspectos didácticos de la modelización (Solar, Azcárate y Deulofeu, 2009).

El interés por desarrollar procesos matemáticos en la enseñanza de las matemáticas no es nuevo: como se constató en Solar et al. (2012) se puede hacer una extensa lista de procesos definidos como propios de las matemáticas (representar, argumentar, demostrar, clasificar, analizar, resolver, conjeturar, razonar, visualizar, calcular, etc.); en el trabajo citado se sostiene que si bien los procesos han estado presentes en algunas propuestas curriculares de matemáticas, tales como la propuesta curricular del NCTM (2003), no han tenido un papel destacado en comparación con los contenidos; la mayoría de los currículos tienen como punto de partida los contenidos matemáticos, y los procesos aparecen de manera implícita en las orientaciones didácticas de cada contenido.

En la perspectiva de competencia matemática del marco teórico del informe PISA, se ha puesto el interés en identificar el nivel de dominio de una competencia. En el marco original de PISA (OCDE, 2003) se planteaban "grupos de competencia» (reproducción, conexión y reflexión) para identificar el nivel de complejidad de una actividad. En el marco actualmente vigente (OCDE, 2012), no aparecen estos grupos de competencia y se ha puesto el acento en los niveles de complejidad de cada una de las competencias matemáticas. La complejidad en la modelización depende de criterios tales como si el modelo viene dado o se debe elaborar, y en la complejidad de los supuestos, relaciones y restricciones para que el modelo satisfaga al problema. Nosotros hemos adaptado esta idea y hemos reformulado el planteamiento para desarrollarlo en la competencia de modelización. ¿¿De qué manera se progresa en la competencia de modelización? ¿Cuáles son los criterios que hay que considerar para estudiar el progreso de los estudiantes?

El propósito general de este trabajo es estudiar la modelización como una competencia matemática. Para ello partimos de la base de que, si bien las competencias matemáticas son transversales a los contenidos, estos sustentan el desarrollo de estas, y entendemos que no se puede caracterizar una competencia sin estar asociada a un tema matemático. Nuestra propuesta se ha desarrollado en el tema de «interpretación de gráficas funcionales», incorporando la noción de proceso como el componente diferenciador, de tal manera que la competencia de modelización se conforma por unos procesos que corresponden a este tema matemático concreto. Si estuviéramos en otro tema, algunos de los procesos que conforman la competencia de modelización podrían cambiar. Estas condiciones hacen que la competencia de modelización sea transversal a los contenidos, pero los procesos propios que la conforman dependan del tema matemático en juego. 
Para estudiar la modelización como una competencia hemos elaborado un Modelo de Competencia Matemática (MCM) que relaciona contenidos, procesos y niveles de complejidad de la actividad. En otros trabajos (Espinoza et al., 2008) se ha utilizado el MCM para realizar un diseño curricular basado en competencias matemáticas; también se ha puesto a prueba para estudiar la argumentación como una competencia (Soplar et al., 2012), y además este modelo se ha utilizado para la formación de profesores de primaria (Solar, Rojas, Ortiz y Ulloa, 2012).

Para este artículo, estudiamos la aplicación del MCM a la competencia de modelización, basándonos en tareas, procesos y nivel de complejidad. La pregunta que abordamos es «¿De qué manera se relacionan las tareas, los procesos y la complejidad en el desarrollo de la competencia de modelización?». En el desarrollo del artículo nos centraremos en describir cómo se caracterizan las tareas y los procesos en la interpretación de gráficas funcionales, y de qué manera, articulando estos dos componentes, se puede determinar el nivel de complejidad de una actividad matemática en el aula; con ello se describirá la función didáctica de la modelización tanto en la planificación de una secuencia didáctica como para el análisis de la modelización en el aula.

\section{MARCO TEÓRICO}

\section{Modelización matemática}

Existe una variedad de descripciones sobre el término modelización. La propuesta de Penrose (1978; extraído de Houston, 2007) es una de las pioneras que describe los pasos en el proceso de modelización. Posteriormente han seguido propuestas con la misma base pero que destacan otros aspectos, como el modelo de la situación real (Maaß, 2006) y la relación entre la teoría y los datos en el proceso de modelización (Blomhøj y Højgaard, 2003). Desde un enfoque evaluativo Houston (2007) caracteriza criterios para evaluar las fases de la modelización en los estudiantes. En términos generales, la modelización se presenta en la literatura como las fases para resolver un problema proveniente de una situación real por medio de un modelo matemático (Maßß, 2006). Se inicia generalmente con una situación extramatemática, se simplifica a un modelo real y se matematiza para obtener un modelo matemático; se resuelve dentro del modelo y se interpreta la solución de modo coherente con la situación inicial; finalmente se evalúa si responde a la situación original. El sistema se concibe como dinámico y cíclico, pues en este se producen continuas transformaciones para mejorar la interpretación y predicción del fenómeno.

Para un tratamiento didáctico, esta manera de entender la modelización es insuficiente dado que se centra en las fases para resolver un problema, y no trata sobre el desarrollo de modelización en el aula de matemáticas. Para caracterizar la competencia de modelización, además de considerar las fases de modelización de Maßß (2006), nos hemos sustentado en otros dos enfoques que permitan darle una función didáctica: los trabajos conducidos por de Lange (1987), sobre matematización en que se caracteriza matematización horizontal con procesos matemáticos para transitar del mundo real al mundo matemático, y matemátización vertical con procesos matemáticos propios del mundo matemático. En el marco teórico actual de PISA (OECD, 2012) se han realizado modificaciones en la línea de dar una mayor relevancia a la modelización. Uno de los cambios desde el marco del 2003 ha sido modificar la competencia «modelizar» a «matematizar», pero el cambio más significativo ha sido destacar los procesos de modelización -formular, emplear, interpretar- en la caracterización de mathematical literacy, ${ }^{1}$ en que se describe de qué manera las competencias matemáticas se desarrollan en cada una de estos

1. Mathematical literacy se ha traducido al castellano en el informe PISA (OECD, 2003, 2012) como «Competencia matemática». Pero, para evitar confusiones con nuestro significado de competencia matemática, preferimos mantener el término en su versión original. 
procesos de la modelización, incluida la matematización. Este cambio en el marco teórico de PISA vincula la actividad matemática escolar de forma directa con los procesos de modelización.

Esta visión de modelización es más amplia que la anterior expuesta, dado que se enfoca hacia la enseñanza de la matemática y no solo al tratamiento de un problema, como es el caso de las fases de modelización.

Los trabajos sobre modelización emergente (Gravemeijer y Doorman, 1999; Gravameijer, 2007) constituyen un enfoque de particular interés para atribuir una función didáctica a la competencia de modelización. El término emergente se refiere tanto al carácter del proceso por el cual los modelos surgen en la educación matemática realista, como al proceso por el cual estos modelos apoyan la aparición de los modos matemáticos formales de saber. En primer lugar, el modelo empieza a destacar como un modelo de las estrategias informales de los estudiantes, y con el tiempo el modelo se convierte, de manera gradual, en una entidad propia y empieza a servir como un modelo para un razonamiento matemático más formal. Con relación a esto, se pueden distinguir cuatro tipos o niveles de actividad matemática:

1. Actividad en el entorno de trabajo, en que las interpretaciones y soluciones dependen de la comprensión de cómo actuar en un escenario cualquiera (a menudo fuera de la escuela).

2. Actividad de referencia, en que los modelos de hacen referencia a una actividad concreta del aula de matemáticas.

3. Actividad general, en que los modelos para obtienen su significado desde un marco de relaciones matemáticas.

4. El razonamiento matemático formal, que ya no depende del apoyo de modelos para la actividad matemática.

Estos cuatro niveles de actividad ilustran que los modelos, inicialmente, están ligados a la actividad en un escenario específico e implican imágenes de la situación específica; en el nivel de referencia, los modelos se basan en la comprensión de los estudiantes y escenarios empíricamente reales. La actividad general empieza a surgir cuando los estudiantes comienzan a decidir sobre las relaciones matemáticas que están implicadas. Como consecuencia, el modelo se libera de su dependencia de imágenes específicas de la situación, y gradualmente se desarrolla en un modelo que obtiene su significado de relaciones matemáticas que están siendo interpretadas en el proceso. La transición desde modelo de a modelo para coincide con una progresión desde un razonamiento matemático informal a uno de carácter formal que se entrelaza con la creación de una nueva realidad. Por consiguiente, la transición modelo de a modelo para no está ligada a las manifestaciones específicas del modelo, sino al pensamiento del estudiante; mientras que el término modelo de se refiere a una actividad en un escenario específico, el término modelo para se refiere a un marco de relaciones matemáticas (Gravemeijer, 2007).

En el MCM se han considerado los tres enfoques de modelización tratados anteriormente. Las fases de modelización (Maßß, 2006) son la base para entender los procesos de modelización. El enfoque de matematización, al ser una visión más amplia, da sentido al hecho de que existan cuatro componentes, que en su conjunto permiten darle una función didáctica a la competencia de desarrollar la modelización en el aula de matemáticas. La modelización emergente sustenta un cambio en el MCM para la competencia de modelización. Dada la importancia que tiene la caracterización del nivel de abstracción del modelo matemático, hemos agregado un componente más en el MCM para la competencia de modelización, que articula las fases de modelización (Maßß, 2006) con los niveles de la actividad en el enfoque de modelización emergente (Gravameijer, 2007). A este componente lo hemos denominado «fases de modelización», ya que cumplen las características de ser un proceso cíclico de modelización.

Respecto al tema matemático en juego, a las funciones se le puede atribuir diferentes significados, tales como: una regla o correspondencia, una máquina que toma una entrada y entrega la correspon- 
diente salida o una dependencia entre variables. Cada uno de estos significados se puede interpretar como un modelo matemático. Al introducir las funciones desde la interpretación de gráficas funcionales, por el tipo de tareas matemáticas que se ponen en juego, tales como interpretar gráficas o traducir de una tabla a una gráfica, el modelo matemático que emerge es el de dependencia de variables. Este modelo se puede representar de diferentes maneras: expresión numérica del modelo (tabla numérica), expresión gráfica, expresión verbal, y expresión algebraica.

\section{Modelo de competencia matemática (MCM)}

El modelo de competencia matemática (MCM) que hemos propuesto se sustenta en la perspectiva funcional de las matemáticas, mathematical literacy (OECD, 2003 y 2012). En concreto, el MCM para la competencia de modelización se conforma en tres dimensiones:

Competencia matemática: basándonos en las propuestas que consideramos más relevantes de competencias matemáticas (Abrantes, 2001; NCTM, 2003; Niss, 2002; Niss y Højgaard; 2011; OECD, 2003; OECD; 2012), acordamos elegir y optar por procesos matemáticos nucleares que denominamos competencias matemáticas, las cuales organizan y articulan el currículo de matemáticas. Para que las competencias sean articuladoras tienen que cumplir cuatro criterios:

- Integración de procesos: integrar una serie de procesos matemáticos específicos vinculados a una competencia matemática.

- Relevancia matemática: ser significativas para la actividad matemática escolar.

- Transversalidad: las competencias se caracterizan por ser transversales a los objetos matemáticos.

- Afinidad: la transversalidad recién definida está condicionada por relaciones de afinidad entre competencias y objetos matemáticos; en particular los procesos tienen distintos niveles de afinidad con el conocimiento matemático puesto en juego.

Organizaciones matemáticas: pese a que los contenidos matemáticos no se constituyen como el elemento estructurante del MCM, se hace necesario articularlos con los procesos o competencias matemáticas específicas. En particular se utilizaron las bases de la Teoría Antropológica de lo Didáctico (TAD) (Chevallard, 1999), en la cual los contenidos matemáticos se estructuran en términos de organizaciones matemáticas, las cuales permiten establecer un núcleo de relaciones entre contenidos, desatomizándolos y permitiendo así una articulación con las competencias matemáticas. Una organización matemática nace como respuesta a un tipo de cuestiones problemáticas y está constituida por cuatro categorías de elementos: tipos de tareas, elementos técnicos, tecnológicos y teóricos. La utilización de la TAD ha servido para caracterizar los contenidos matemáticos, por dos razones. En primer lugar, las tareas matemáticas permiten ser articuladas con los procesos matemáticos para el desarrollo de una competencia matemática específica en el aula. Una segunda razón tiene relación con la noción de variable didáctica proveniente de la Teoría de Situaciones Didácticas de Brousseau (1997), en que la complejidad de las técnicas que utiliza un resolutor depende de las condiciones de realización -o valores de la variable- de una tarea matemática. Este criterio es considerado en la tercera dimensión del MCM que se describe a continuación.

Niveles de complejidad: el progreso de la competencia se determina en términos de la complejidad de la actividad, que depende tanto de las tareas como de los procesos que la conforman. La expresión nivel de complejidad se adopta de los grupos de competencia del marco inicial de PISA (OCDE, 2003) basados en los trabajos desarrollados por de Lange (1995). Sin embargo, en PISA se definió cada nivel de complejidad (reproducción, conexión, reflexión) sin que se presentaran criterios comunes que permitieran identificar de qué elementos depende la complejidad. En cambio, en el MCM los niveles de complejidad de una actividad se determinan con elementos comunes, y están en función de las tareas matemáticas y sus 
condiciones de realización (variables didácticas) y de los procesos específicos que conforman una competencia matemática. A partir de nuestros estudios empíricos (Solar et al., 2012) se ha visto la conveniencia de agregar un nivel más entre conexión y reflexión, el cual hemos denominado generalización, resultando así cuatro niveles de complejidad: reproducción, conexión, generalización y reflexión.

En la figura 1 se puede apreciar la relación entre los componentes del MCM, cuyo elemento central son los procesos matemáticos que permiten organizar y articular el currículo de Matemáticas por medio de los cuatro aspectos clave ya mencionados: integración, relevancia, transversalidad y afinidad.

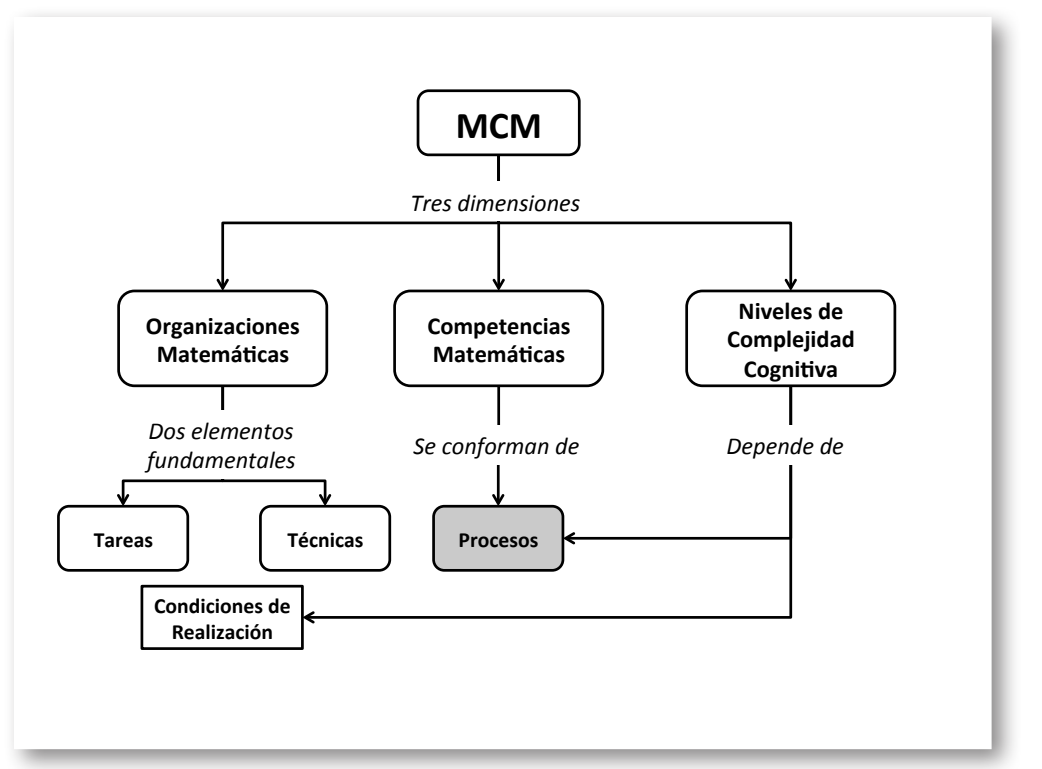

Fig. 1. Modelo de competencia matemática (MCM).

Para el caso particular de la competencia de modelización, se ha agregado una cuarta dimensión denominada Fases de modelización. Para determinar el nivel de abstracción de los modelos matemáticos en juego, se ha considerado el marco de modelización emergente de Gravameijer (2007), que sitúa la actividad en uno de los cuatro niveles de modelización: entorno de trabajo, referencial, general o formal.

\section{METODOLOGÍA}

La experiencia de la cual se obtuvieron los datos de esta investigación se realizó en Santiago de Chile, dentro del marco de proyecto LEM (lectura, escritura y matemáticas) del centro Felix Klein de la Universidad de Santiago de Chile. En el segundo semestre del 2007, un grupo de cuatro profesoras de octavo básico (de 13 a 14 años) implementaron la unidad didáctica denominada "Analizando y construyendo gráficos», diseñada por el equipo LEM (Espinoza, Barbé, Cerda, Waisman, Ruiz y Gálvez, 2006). Esta unidad tuvo como propósito introducir las funciones por medio de la interpretación y construcción de gráficas funcionales y constó de tres etapas: la primera estaba asociada al sistema de representación cartesiano y centrada en la ubicación de puntos en el plano; en la segunda etapa se estudió la noción de dependencia de variables, y la tercera etapa se centraba en la interpretación y construcción de gráficas, particularmente en la inclinación de la curva. Desde el punto de vista de la modelización hay dos modelos matemáticos en juego que permiten fundamentar las diferentes tareas matemáticas que se abordaron en la unidad: en la primera etapa el modelo es el sistema de coordenadas cartesianas, y en la segunda y tercera etapa se trató del modelo de dependencia de variables. 
La unidad didáctica fue analizada con un carácter descriptivo, por medio del instrumento «matriz de competencia» (Espinoza et al., 2008) que permite caracterizar cada actividad, a partir de la identificación de las tareas matemáticas y de su nivel de complejidad de acuerdo con la competencia de modelización. Este análisis previo dio pie a adaptar la unidad didáctica inicial a las necesidades de la investigación, remplazando algunas actividades por otras que a priori desarrollaran niveles altos de complejidad para la competencia de modelización. Una vez realizados estos cambios, se implementó la unidad didáctica.

De la unidad didáctica adaptada, para la recogida de datos se han seleccionado seis tareas matemáticas, que se han clasiificado en tres etapas.

- Etapa 1: identificar sistemas de referencia, construir sistemas de referencia.

- Etapa 2: identificar variables, estudiar dependencia entre variables.

- Etapa 3: traducir representaciones, interpretar gráficas.

Para este estudio se eligió el caso del aula de matemáticas de la profesora Valentina: es decir, la terna alumnos-decente-actividad matemática, en vez de recoger datos entre las cuatro profesoras. La decisión se justifica porque los datos recogidos en las observaciones de clases de Valentina permitían profundizar en los propósitos de la investigación en un solo caso, mientras que si se hubieran recogido datos de varios casos no se hubiera podido ahondar de la misma manera en los datos recogidos. Por esta razón se registraron cinco clases para tener un proceso más completo en el desarrollo de la unidad didáctica con énfasis en registrar las actividades incorporadas. En particular se escogió el caso de la clase de Valentina porque la dinámica de su clase promovía de manera especial la interacción entre la profesora y los 35 alumnos que formaron el grupo de octavo básico. Valentina promovió que los modelos matemáticos estudiados emergieran en conjunto con los estudiantes, característica significativa para el estudio de la modelización en el aula. El análisis del caso tiene como propósito caracterizar las relaciones entre los diferentes componentes: tareas, procesos y complejidad.

Seguimos el transcurso de la implementación de la unidad didáctica con una estrategia de observación no participante durante cinco sesiones. Por medio de grabaciones en video, consideramos los registros enfocados a la interacción entre la profesora y los estudiantes. Finalmente transcribimos las cinco sesiones grabadas.

Si bien las tareas matemáticas en gráficas funcionales se han podido caracterizar desde la literatura (Leinhardt, Zaslavsky y Stein, 1990), en el caso de los procesos matemáticos que conforman la competencia de modelización no se tienen suficientes antecedentes teóricos que permitan caracterizar estos procesos. Por ello, hemos aplicado una metodología inductiva para caracterizar los procesos.

De las cinco sesiones, se han seleccionado aquellos episodios en los que hay interacción entre la profesora y los estudiantes y que muestran una mayor riqueza en los procesos de modelización. La unidad de análisis correspondió a cada una de las acciones del episodio, tanto de Valentina como de los estudiantes. Cada acción se caracterizó con un indicador de proceso asociado a la competencia. Esta estrategia siguió un criterio de comparación constante entre los procesos que emergieron de los episodios analizados y concluyó con la caracterización de un listado de procesos asociados a la competencia de modelización. Dado el carácter innovador de estos procesos, se aplicaron dos instancias de validación, una interna en que se analizó el significado de cada uno de estos procesos, y si su conjunto era coherente con una secuencia de modelización, y una instancia posterior de validación externa en que dos jueces externos a la investigación, en calidad de expertos, discutieron con el investigador la pertinencia y fiabilidad de los procesos elaborados mediante los siguientes criterios: valoración del proceso de construcción de las definiciones, coherencia entre el conjunto de procesos, diferencia entre procesos y tareas, y pertinencia de los procesos de modelización sobre el contenido abordado. La participación de los jueces no se limitó solo a validar los procesos, sino que además contribuyó a solventar los marcos teóricos de la modelización. 
Por otra parte, se han caracterizado las fases de modelización en interpretación de gráficas. Si bien las fases de modelización tienen respaldo en la literatura (Maßß, 2006), estas son generales y no están enfocadas a un contenido. Por tanto, desde una perspectiva inductiva, se ha repetido la estrategia de comparación constante para identificar las fases de modelización en los episodios. En vez de tomar como unidad de análisis cada acción en el aula, las unidades correspondieron a un conjunto de acciones consecutivas que apuntaran a una de las fases de modelización.

En este artículo nos centramos en dos de las cinco sesiones registradas, en las sesiones 1 y 5 , dado que estas actividades fueron incorporadas a la unidad didáctica para promover especialmente la modelización matemática. Para el análisis de datos se ha seleccionado un episodio de cada sesión, ya que en la interacción entre profesora y estudiantes de ambos episodios surgen varios de los procesos propios de la competencia de modelización.

\section{ANÁLISIS Y RESULTADOS}

El estudio de la competencia de modelización se ha organizado en cuatro apartados: en el primero se caracteriza la competencia de modelización: en el segundo y tercero se realiza el análisis de episodios de la primera y quinta sesión respectivamente, y por último, en el cuarto, se discute el progreso de la competencia de modelización a partir de la comparación entre los dos episodios.

\section{Caracterizar la competencia de modelización}

En la investigación se han caracterizado los cuatro componentes de la competencia de modelización: tareas, procesos, fases de modelización y nivel de complejidad.

Tareas: las tareas matemáticas provienen de la unidad didáctica inicial complementadas con otras a partir del análisis previo de la unidad didáctica. Se detallan en la figura 3.

Procesos: en la figura 2 se muestra la caracterización de procesos que conforman la competencia de modelización, en la primera columna se describe el proceso seńalado en cursiva, y en la segunda columna un ejemplo en la unidad didáctica de interpretación de gráficas funcionales.

\begin{tabular}{|c|c|}
\hline Procesos & Ejemplo en la unidad didáctica \\
\hline Identificar o describir las características de un modelo. & $\begin{array}{l}\text { Describir que las coordenadas en un sistema cartesiano son numéricas y } \\
\text { tiene punto de origen }(0,0) \text {. }\end{array}$ \\
\hline $\begin{array}{l}\text { Interpretar el modelo o su expresión (gráfica, tabla, expresión } \\
\text { verbal o algebraica). }\end{array}$ & $\begin{array}{l}\text { Interpretar la gráfica de la temperatura de ciudades ordenadas de norte a } \\
\text { sur. }\end{array}$ \\
\hline $\begin{array}{l}\text { Validar características del modelo: Aseveraciones que validen o } \\
\text { refuten las descripciones de las características o la } \\
\text { interpretación del modelo. }\end{array}$ & $\begin{array}{l}\text { Ejemplo Profesora: cantidad, él dijo por ejemplo, si en un minuto se llena } \\
10 \mathrm{~cm} .\end{array}$ \\
\hline $\begin{array}{l}\text { Construir la expresión de modelo (sistema de referencia, gráfica, } \\
\text { tabla, etc.). }\end{array}$ & Elaborar un gráfico a partir de los datos. \\
\hline Identificar las propiedades del modelo matemático. & Identificar las variables. \\
\hline $\begin{array}{l}\text { Aplicar el modelo y la expresión correspondiente a la situación } \\
\text { problemática. }\end{array}$ & $\begin{array}{l}\text { Establecer si hay dependencia entre dos variables estudiando la relación } \\
\text { entre sus datos. }\end{array}$ \\
\hline $\begin{array}{l}\text { Validar el modelo o refutar ( representaciones y/o propiedades } \\
\text { del modelo). }\end{array}$ & $\begin{array}{l}\text { Contrastar la coherencia de la construcción de un gráfico con la expresión } \\
\text { verbal. }\end{array}$ \\
\hline $\begin{array}{l}\text { Reflexionar sobre la modelización: el modelo, las fases de } \\
\text { modelización y su aplicación como solución a la situación } \\
\text { problemática. }\end{array}$ & Analizar las limitaciones de un sistema de referencia con letras y números. \\
\hline
\end{tabular}

Fig. 2. Procesos de la competencia de modelización. 
Ocho procesos conforman la competencia de modelización, que se pueden asociar con las cinco fases de modelización presentadas por Maßß (2006): simplificación, matematización, trabajo matemático, interpretación y validación. Caracterizar el modelo es un proceso que generalmente ha emergido en la fase de simplificación al identificar las propiedades del modelo real. El proceso de interpretar el modelo comprende acciones tanto hacia el modelo como a su expresión (gráfica, tabla). Las acciones de construcción se asocian al proceso de obtención de la expresión del modelo. Estos dos procesos han emergido generalmente en la fase de matematización (paso del modelo real al modelo matemático), y en menos ocasiones en la fase de interpretar la solución que proviene de modelo matemático en la situación original. El proceso de identificar propiedades del modelo se enfoca hacia las propiedades del modelo matemático; este se ha dado en escasas ocasiones y en la fase de matematización. El proceso de aplicar el modelo, que implica utilizar de manera explícita el modelo matemático y la expresión correspondiente a la situación problemática, se ha dado en escasas ocasiones y en la fase de interpretar la solución. Los dos procesos de validación (de las características del modelo y del modelo) emergen en fases y con frecuencias distintas. Las acciones asociadas a validar las características del modelo se han dado frecuentemente entre la simplificación y la matematización; en cambio, la validez del modelo ha emergido en escasas acciones coincidiendo con la fase de validación. Del mismo modo, el proceso de reflexionar acerca de la modelización consiste en acciones críticas sobre el modelo, las fases de modelización y la aplicación de la solución a la situación problemática.

El desarrollo de los ochos procesos en su conjunto se considera como la construcción del modelo, tal como el de dependencia de variables. En la interpretación de gráficas, construir el modelo significa: caracterizar sus elementos, trabajar con sus expresiones asociadas, interpretarlo, validarlo y aplicarlo; es decir, cada uno de los procesos contribuye a la construcción del modelo. Este significado es diferente del tradicional, que entiende la elaboración de la expresión algebraica o de la gráfica como la construcción del modelo. En esta investigación hemos seguido a Gravemeijer (2007), quien señala que el modelo se entiende más como un concepto que como un modelo propiamente dicho.

Fases de modelización: de acuerdo con el análisis de los episodios, se han identificado cinco focos en la interpretación de gráficas. Modelo real, variables, valores de la variable, modelo matemático y expresión del modelo matemático. Estos cinco focos, a diferencia de los procesos, se identifican en un intervalo continuo de acciones que centran la atención en uno de los cinco aspectos mencionados. Estos focos, que son parte de la interpretación de gráficas, se pueden asociar a las fases de modelización señaladas por Maaß (2006). La correlación entre las fases y los focos de modelización se da de la siguiente manera: en la fase de simplificación se analiza el modelo real, y en la fase de matematización se pasa hacia el mundo matemático, dado que entran en juego las variables. La fase de trabajo con el modelo se focaliza en el modelo matemático y en sus expresiones; la fase de interpretar el modelo matemático se focaliza en la interpretación de su expresión y de las variables. Finalmente, en la fase de validación, las acciones se centran principalmente en validar el modelo matemático, aunque también se validan los cuatro focos restantes (expresión del modelo, variables y sus valores). Si se refuta alguno de estos focos se vuelve a un foco anterior. Dada esta correspondencia entre las fases de modelización y los cinco focos, al componente que agrupa estos aspectos se le ha atribuido el nombre de fase de modelización.

Para analizar este componente se adoptó la perspectiva de modelización emergente (Gravemeijer y Doorman, 1999). De acuerdo con el diseño heurístico de modelización emergente, el modelo comienza a destacar como un modelo de las estrategias informales de los estudiantes. Con el tiempo, el modelo gradualmente se convierte en una entidad propia y empieza a servir como un modelo para un razonamiento matemático más formal.

Nivel de complejidad: se han identificado cuatro niveles de complejidad: reproducción, conexión, generalización y reflexión. 
Competencia de modelización: Enunciados todos los elementos, en la figura 3 se visualiza la relación entre procesos, tareas, fases de modelización y niveles de complejidad. Muchas tareas se repiten en los niveles, tales como interpretar gráficas, traducir y estudiar la dependencia entre variables, y lo que cambia son los procesos en cada nivel. El modelo puede ser aplicado tanto a la planificación de una unidad didáctica como a su desarrollo en el aula y sirve para relacionar los componentes de la competencia e identificar el nivel de complejidad de una actividad.

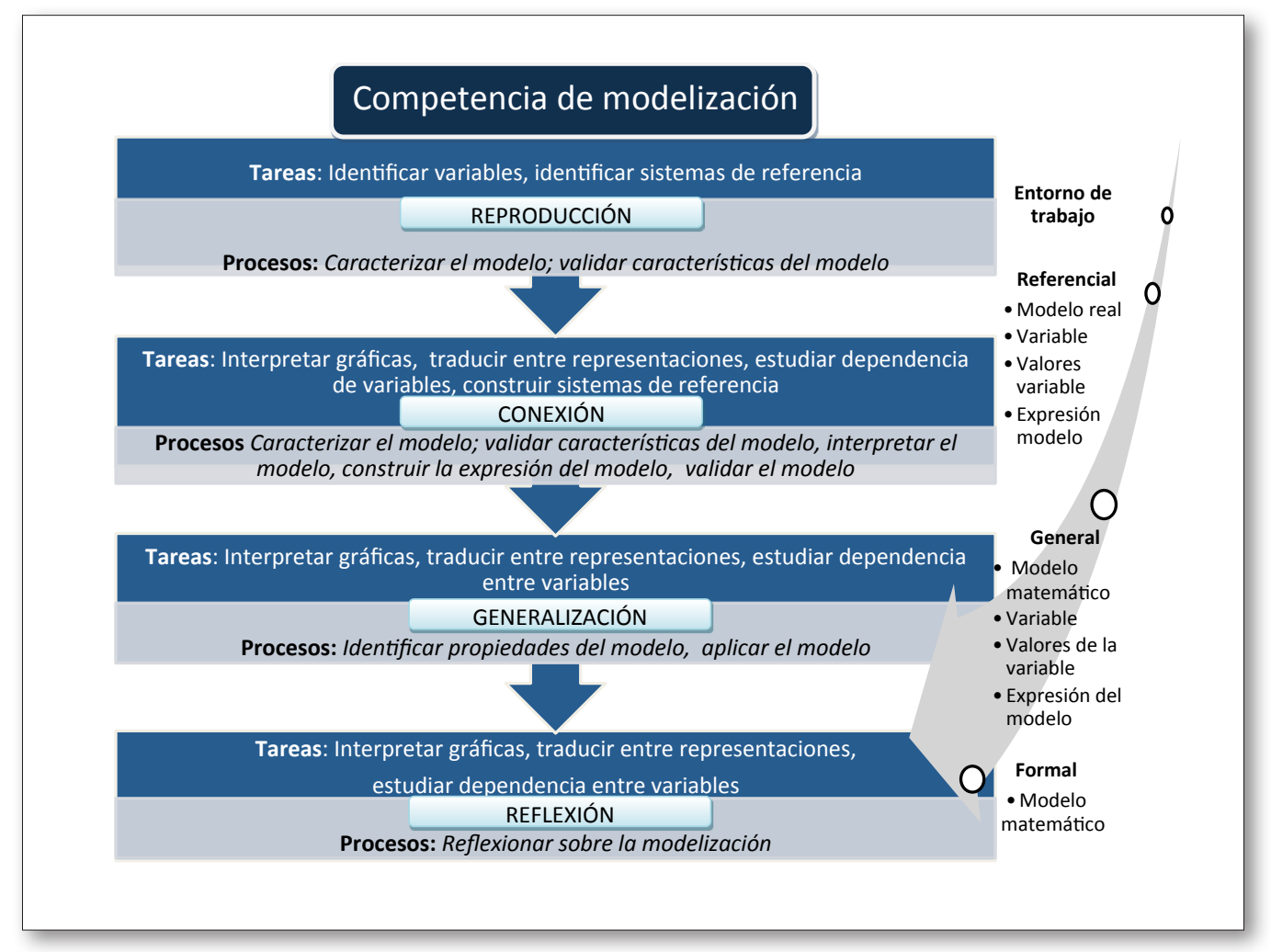

Fig. 3. Competencia de modelización.

En la parte derecha de la figura 3 se ilustra de qué manera se asocian las fases de modelización con los niveles de la actividad. De la relación entre tareas, procesos y fases de modelización se determinan los niveles de complejidad de la actividad: reproducción, conexión, generalización o reflexión.

\section{Análisis de la sesión 1}

La sesión 1 forma parte de la etapa 1, "ubicando puntos en el espacio», de la unidad, que tiene como objetivo desarrollar la noción de sistema de referencia. Se desarrolla en parejas una actividad que consiste en que cada estudiante sitúe dos tesoros en un tablero 6x6 dibujado en su cuaderno de notas, y que no puede ser visto por su compańero. Luego, por turnos, se debe adivinar la ubicación en que el otro jugador situó sus tesoros (figura 4). En el desarrollo de la actividad, los estudiantes construyen diferentes sistemas de referencia, tales como usar filas y columnas, letras y números. En la puesta en común de los sistemas de referencia utilizados, la profesora Valentina estudia con todo el grupo las limitaciones de cada uno de estos sistemas y las condiciones que tiene que cumplir un buen sistema de referencia. Ante ello, Valentina propone un sistema que consiste en cambiar las letras para dejar solo 
números y promueve a los estudiantes a establecer criterios para caracterizar este sistema de referencia. En el episodio seleccionado (figura 5) interviene Ignacio, quien sugiere establecer una regla como criterio, y con la aprobación de Valentina, Ignacio describe la regla para definir un sistema de referencia con números, que vendría a ser un sistema cartesiano (las intervenciones de la profesora se han ennegrecido). Tras ese episodio Valentina introduce las gráficas cartesianas y sus propiedades.

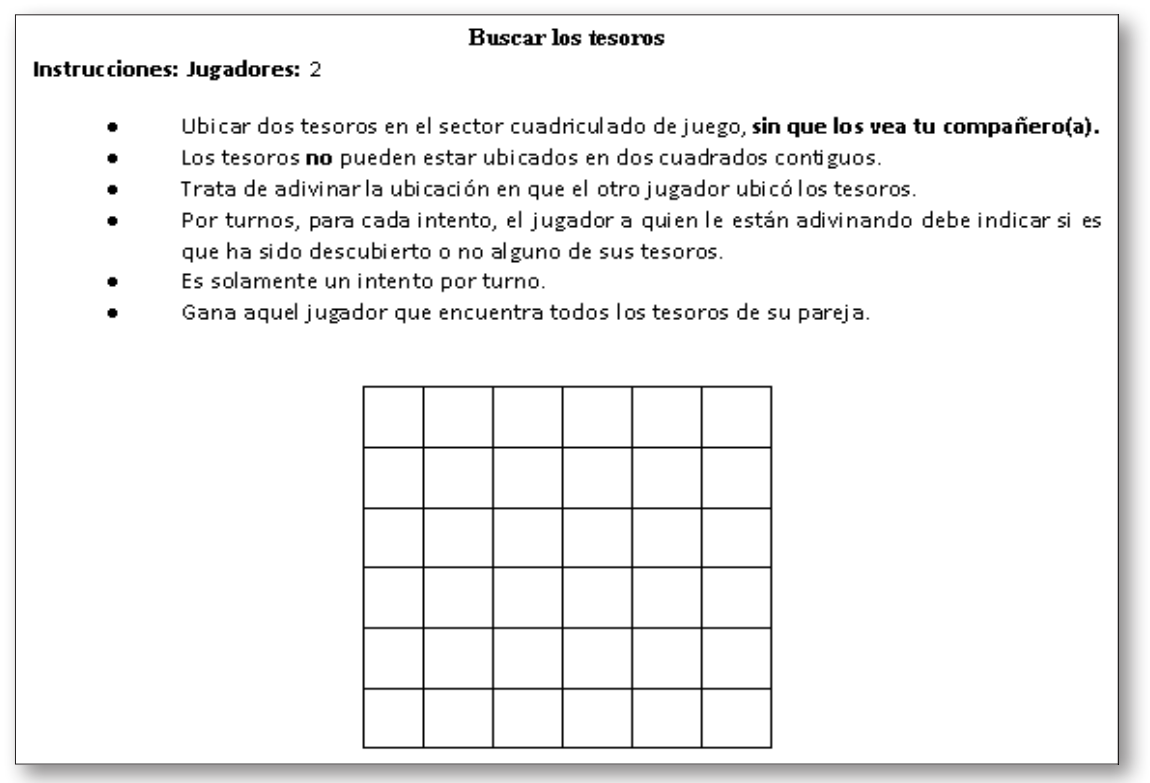

Fig. 4. Actividad sesión 1.

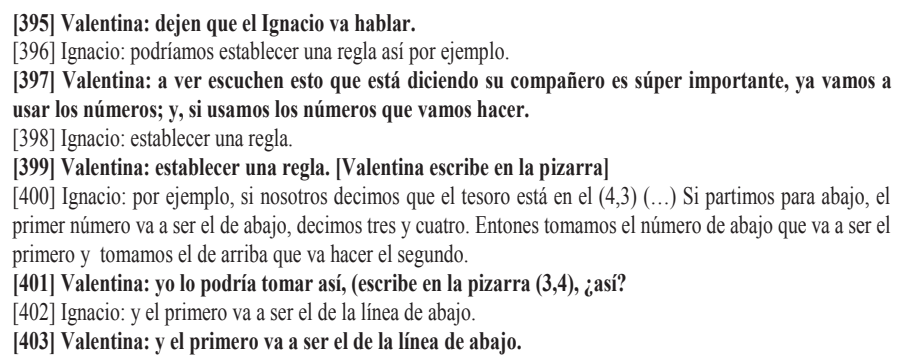

Fig. 5. Episodio sesión 1.

Para este episodio se caracterizan los cuatro componentes de la competencia de modelización:

Tareas: a lo largo de la sesión 1 se desarrolla la tarea de identificar un sistema de referencia; en el episodio, al describir la regla ([400] - [403]), se identifica el sistema cartesiano como el modelo apropiado para describir los puntos en el plano (tesoros en el sector cuadriculado); después de este episodio se construye el sistema cartesiano que corresponde a la tarea matemática de construir un sistema de referencia.

Procesos: para ilustrar los procesos que intervienen se ha elaborado un «mapa de procesos» del episodio. En cada celda se inscribe una parte de la acción o una síntesis de esta que describa el proceso que está emergiendo. El mapa de proceso (figura 6) ilustra los procesos que emergen en el episodio. 


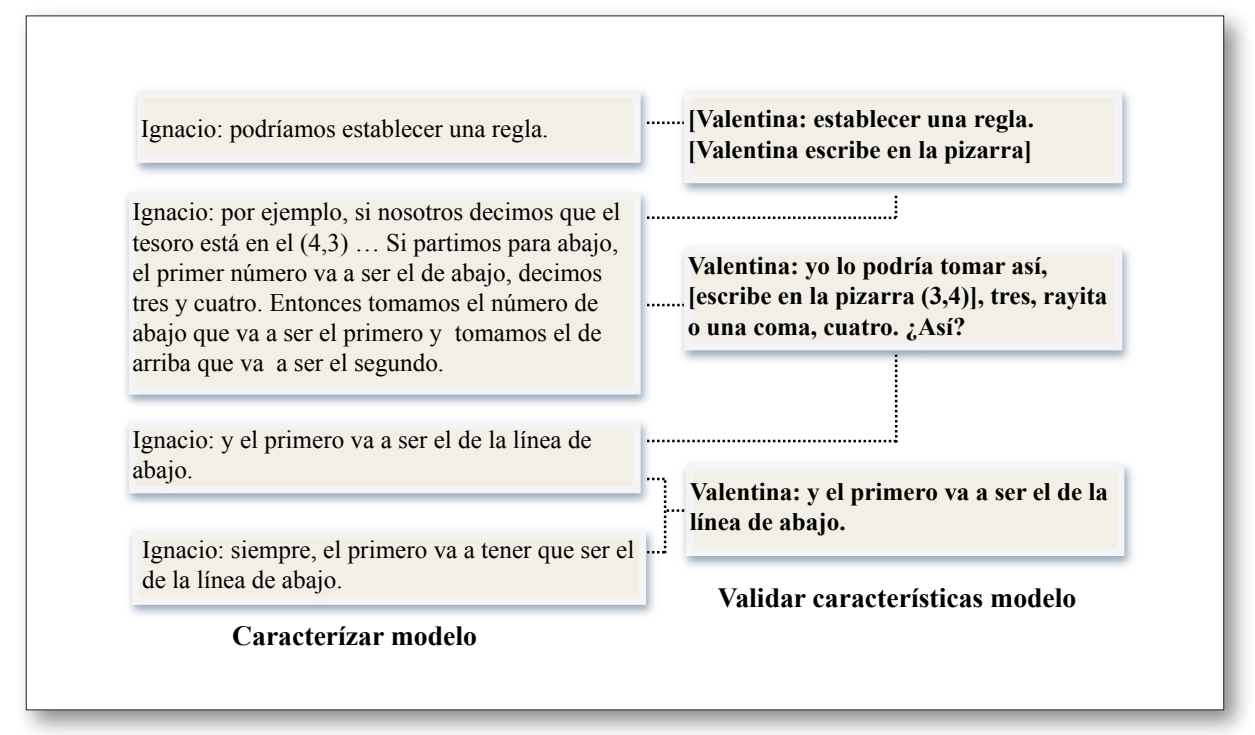

Fig. 6. Mapa de proceso episodio 1.

El modelo que se desarrolla es el sistema cartesiano; el mapa de proceso muestra un ciclo en torno al proceso de caracterizar este modelo. Cuando Ignacio propone la regla, al repetir lo dicho por Ignacio y escribir el punto en la pizarra, Valentina valida su intervención, que también sirve para que Ignacio siga describiendo el sistema de referencia.

Fases de modelización: en la sesión emergieron varios modelos de sistemas de referencia; en particular, en el episodio se aprecia de qué manera está emergiendo el sistema cartesiano, que se termina de desarrollar cuando Valentina introduce las gráficas cartesianas y sus propiedades. Como el modelo sistema cartesiano ha surgido del contexto de la actividad, corresponde a un modelo de la actividad. Esto permite identificar un nivel referencial de la actividad.

Niveles de complejidad: siguiendo el modelo ilustrado en la figura 3, la relación entre tareas, procesos y fases de modelización en este episodio corresponde a un nivel de conexión. En la sesión completa emergen otros procesos pero estos no significan un nivel de complejidad mayor, puesto que el modelo recién ha emergido y aún falta trabajar con el modelo en otras actividades. Por tanto se mantiene un nivel de conexión.

Análisis del episodio de la sesión 5

La actividad «Accidentes automovilísticos» (figura 7) es la última en la secuencia de la unidad didáctica «Analizando y construyendo gráficos». La manera de presentar la actividad no es un contexto habitual para los estudiantes ya que la gráfica, en vez de ser elaborada para una actividad de aula, proviene originalmente de un periódico. La actividad presenta una gráfica que muestra la velocidad promedio de un coche en el momento que tiene un accidente en el transcurso de cinco años. Las preguntas se enfocan a producir una reflexión en los estudiantes en la interpretación de gráficas. Las primeras dos preguntas corresponden a interpretar la gráfica, para luego presentar un párrafo extraído de la noticia real del periódico asociado a la gráfica. En las tres preguntas siguientes, se pide hacer un comentario sobre la interpretación que se realiza de la gráfica en la noticia, valorando si se está de acuerdo con la interpretación del periodista y situando al estudiante bajo distintas interpretaciones de la gráfica. 


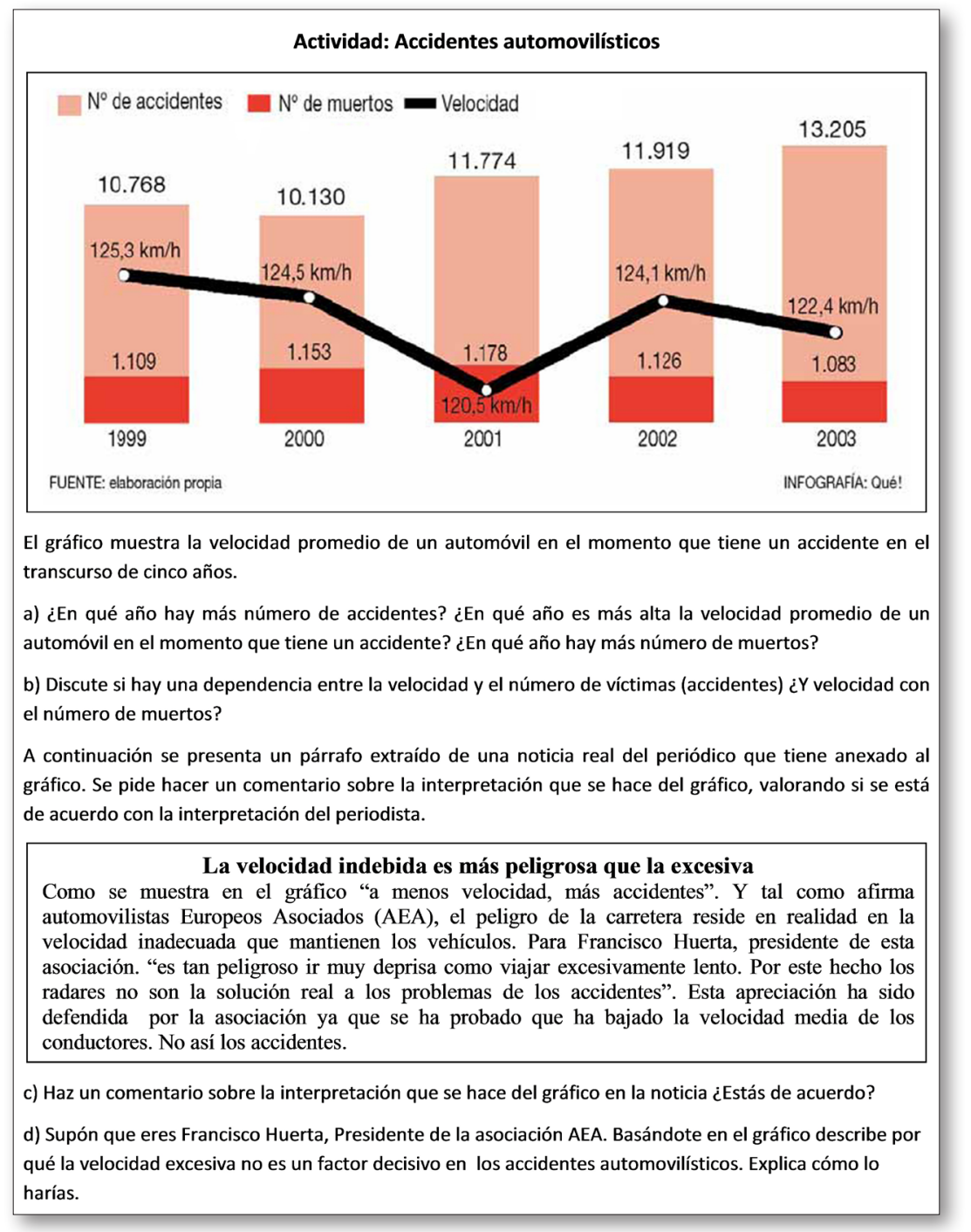

Fig. 7. Actividad sesión 5.

La actividad se inicia centrándose en el modelo real (gráfica de barras). Valentina, si bien induce a los estudiantes reiteradamente a que interpreten la gráfica, se encuentra con dos resultados. Por un lado logra que algunos estudiantes, a partir de la interpretación de la gráfica, determinen que las variables son independientes. Pero por otro lado varios estudiantes responden a la pregunta según ideas previas o espontáneas emergentes, relativas al contexto de la tarea, en detrimento de una focalización en los datos, para determinar si hay dependencia. Por tanto Valentina no logra negociar con el curso una solución consensuada. Ante esto, Valentina cambia la estrategia que utilizaba usualmente para interpretar los datos, que era interpretar directamente la gráfica, y traduce a una expresión numérica (tabla) utilizando el modelo de dependencia entre variables para determinar si la velocidad afecta a los accidentes o muertes. Se conjetura que no hay dependencia porque la relación visual entre los números en la tabla no muestra una relación; a continuación Valentina construye la expresión gráfica del modelo, y a diferencia de antes logra negociar con los estudiantes que no hay ningún tipo de relación de dependencia. La discusión gira en torno a cómo justificar esta afirmación, y es en este punto donde Valentina observa que a los estudiantes les es difícil sostenerse en los datos para justificar que no hay 
dependencia entre las variables. Ello ha implicado un escaso protagonismo de los estudiantes y es Valentina quien finalmente termina desarrollando la actividad.

En las preguntas finales de la actividad se aplican los resultados obtenidos para contrastar las declaraciones en la noticia del periódico. Se analiza un episodio que corresponde a gran parte del desarrollo de la última pregunta (figura 8). Se ha dado inicio a la pregunta, pero los estudiantes no la entienden, muestran confusión y dicen que la interpretación de la noticia es falsa. Valentina acota y explica nuevamente el sentido de la tarea. En la pizarra se mantienen dos frases que se han escrito previamente: «menos velocidad más accidentes» y «a más velocidad menos accidentes», y una tabla en que se han organizado los datos expuestos en la gráfica.

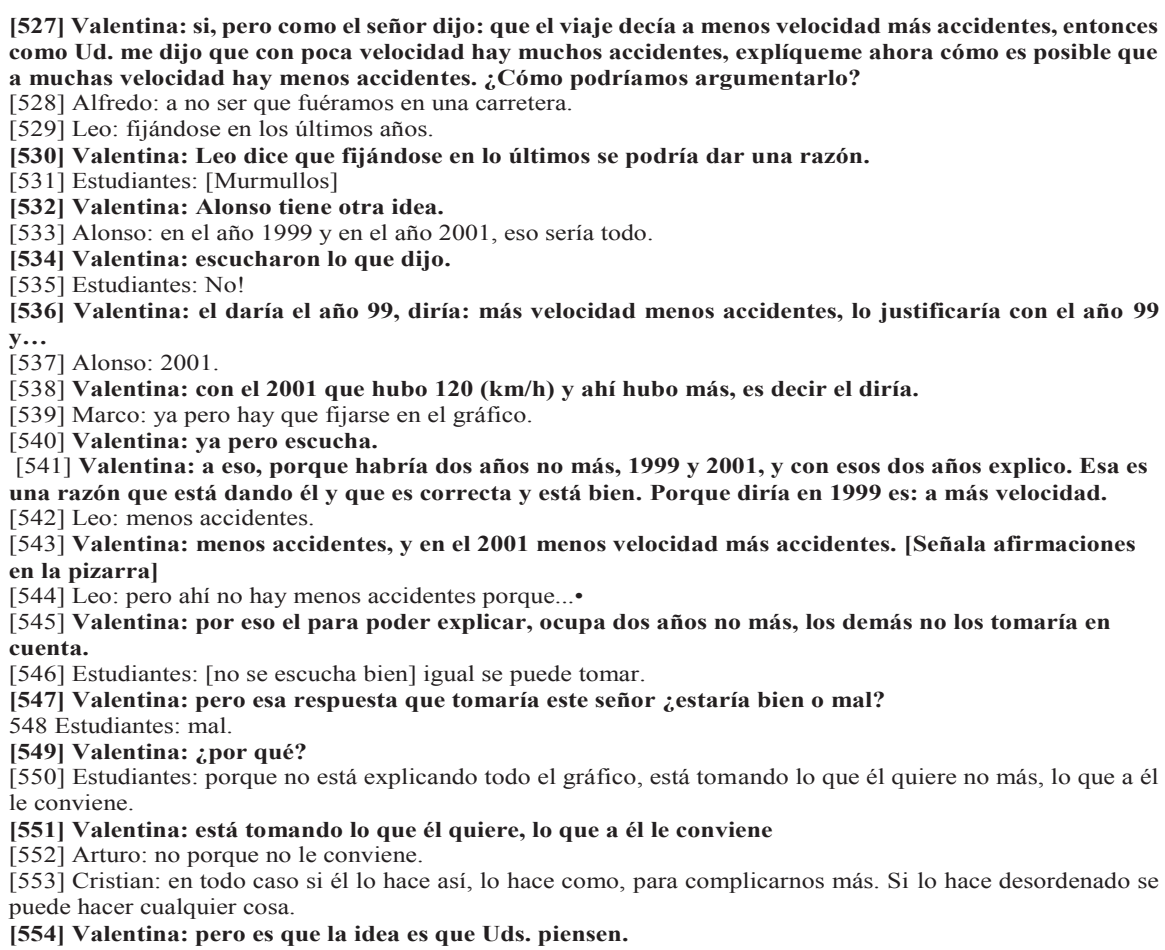

[527] Valentina: si, pero como el señor dijo: que el viaje decía a menos velocidad más accidentes, entonces como Ud. me dijo que con poca velocidad hay muchos accidentes, explíqueme ahora cómo es posible que a muchas velocidad hay menos accidentes. ¿Cómo podríamos argumentarlo?

[528] Alfredo: a no ser que fuéramos en una carretera.

[529] Leo: fijándose en los últimos años.

[530] Valentina: Leo dice que fijándose en lo últimos se podría dar una razón.

[531] Estudiantes: [Murmullos]

[532] Valentina: Alonso tiene otra idea.

[533] Alonso: en el año 1999 y en el año 2001, eso sería todo.

[534] Valentina: escucharon lo que dijo.

[535] Estudiantes: No!

[536] Valentina: el daría el año 99, diría: más velocidad menos accidentes, lo justificaría con el año 99 $\mathbf{y}$...

[537] Alonso: 2001 .

[538] Valentina: con el 2001 que hubo $120(\mathrm{~km} / \mathrm{h})$ y ahí hubo más, es decir el diría.

[539] Marco: ya pero hay que fijarse en el gráfico.

[540] Valentina: ya pero escucha.

[541] Valentina: a eso, porque habría dos años no más, 1999 y 2001, y con esos dos años explico. Esa es una razón que está dando él y que es correcta y está bien. Porque diría en 1999 es: a más velocidad.

[542] Leo: menos accidentes.

[543] Valentina: menos accidentes, y en el 2001 menos velocidad más accidentes. [Señala afirmaciones en la pizarra]

[544] Leo: pero ahí no hay menos accidentes porque...

[545] Valentina: por eso el para poder explicar, ocupa dos años no más, los demás no los tomaría en cuenta.

[546] Estudiantes: [no se escucha bien] igual se puede tomar.

[547] Valentina: pero esa respuesta que tomaría este señor ¿estaría bien o mal?

548 Estudiantes: mal.

[549] Valentina: ¿por qué?

[550] Estudiantes: porque no está explicando todo el gráfico, está tomando lo que él quiere no más, lo que a él le conviene.

[551] Valentina: está tomando lo que él quiere, lo que a él le conviene

[552] Arturo: no porque no le conviene.

[553] Cristian: en todo caso si él lo hace así, lo hace como, para complicarnos más. Si lo hace desordenado se puede hacer cualquier cosa.

[554] Valentina: pero es que la idea es que Uds. piensen.

Fig. 8. Episodio sesión 5.

Al igual que en la sesión 1, caracterizamos los cuatro componentes de la competencia de modelización:

Tareas matemáticas: la tarea principal que se desarrolla a lo largo de la actividad y que se evidencia en este episodio es el estudio de la dependencia de variables.

Procesos: en este episodio se presenta cómo emerge el proceso de reflexionar sobre la modelización, que es uno de los ocho procesos que caracterizan esta competencia. El mapa de proceso (figura 9) ilustra los procesos que emergen en el episodio. 


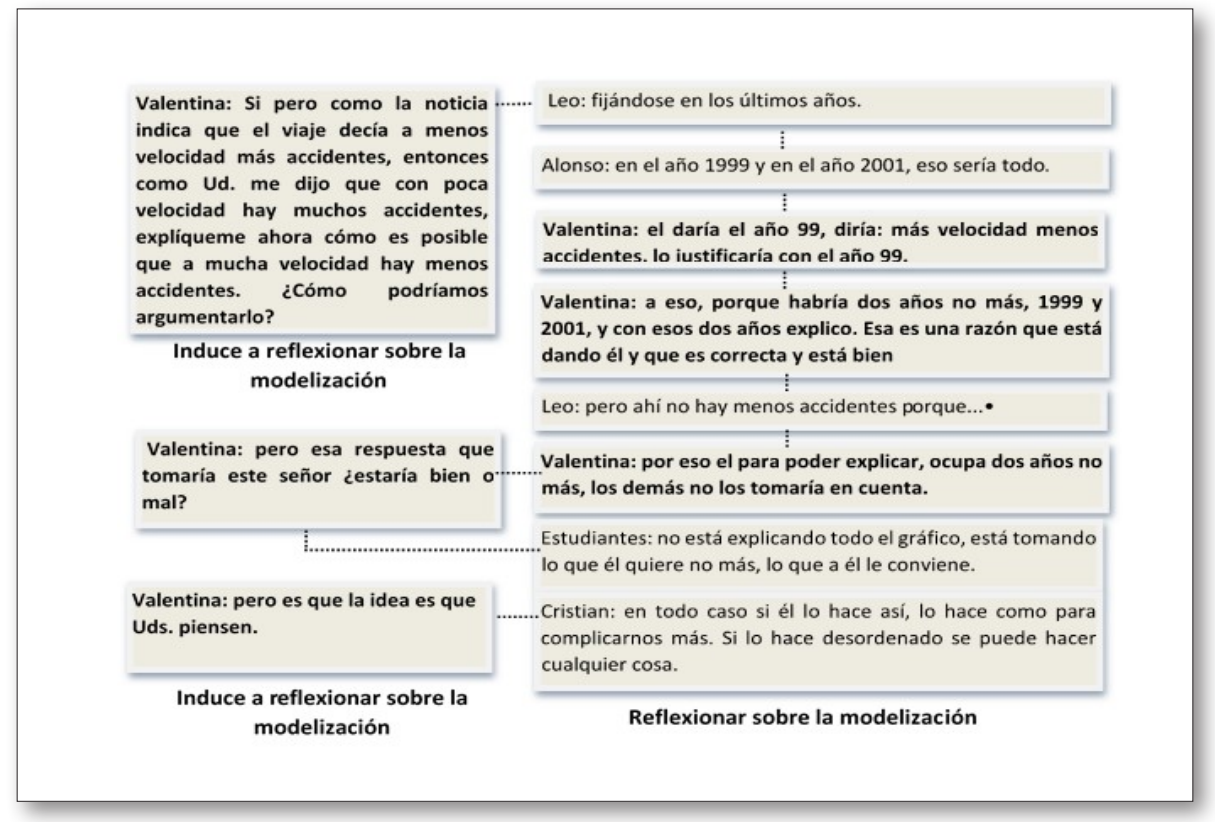

Fig. 9. Mapa de proceso episodio 5.

En un principio Valentina induce a reflexionar sobre la interpretación del modelo de dependencia; esto genera una discusión entre los estudiantes y Valentina, y por tanto en esta interacción el proceso de reflexión sobre la modelización está presente. Si bien este proceso emerge a lo largo del episodio, no significa que se desarrolle completamente. Cristian y otros estudiantes analizan críticamente el proceso de modelización, pero sus argumentos carecen de una justificación a partir de los datos, ya que se sostienen en argumentaciones externas, lo cual se refleja en la última intervención de Valentina, quien sigue induciendo a que razonen sobre el modelo, con el fin de negociar con Cristian y el resto de alumnos una justificación basada en los datos. Valentina induce la reflexión pero los estudiantes no reflexionan como ella esperaba, puesto que es Valentina quien finalmente impulsa las reflexiones y los estudiantes responden brevemente. Por tanto, si bien reflexionar sobre la modelización está presente, este proceso no se desarrolla tal como esperaba la profesora.

Fases de modelización: en el desarrollo de la actividad el modelo de dependencia es conocido por los estudiantes. Si bien es Valentina quien construye la tabla y la gráfica, los estudiantes interpretan de forma fluida estas dos expresiones para determinar la no dependencia entre variables. La familiaridad para los estudiantes en el uso de estas expresiones del modelo se explica porque en sesiones anteriores se ha negociado el modelo de dependencia. En consecuencia, podemos afirmar que el modelo de dependencia entre variables actúa como un modelo para la actividad, que correspondería a un nivel general de la actividad. No es un nivel formal puesto que en esta unidad didáctica aún no se estudia formalmente la noción de función y en consecuencia tampoco la expresión algebraica.

Nivel de complejidad: en el episodio se constata que el proceso de reflexionar sobre la modelización solamente emerge de manera inicial y no se desarrolla completamente. Por tanto, al aplicar el MCM se identifica un nivel de complejidad de generalización en el desarrollo de la actividad en el aula.

En la figura 10 se identifican las tareas y los procesos que se esperan en los dos episodios analizados, y se comparan los niveles de complejidad esperados y desarrollados. El nivel de complejidad esperado está en función de los procesos y tareas, en cambio el nivel de complejidad desarrollado también depende de las fases de modelización. 


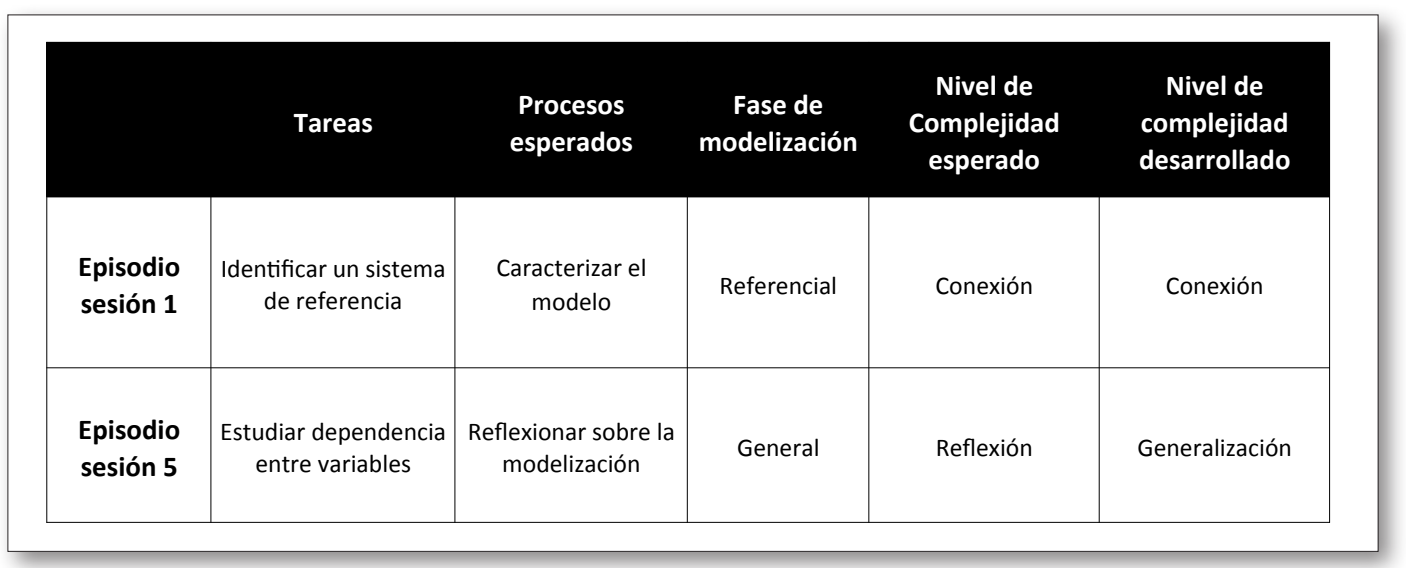

Fig. 10. Comparación de los niveles de complejidad en los episodios.

En el episodio de la sesión 1 el nivel de complejidad esperado coincide con el desarrollado, dado que emergieron los procesos que se esperaban y el nivel referencial de las fases de modelización apunta a conexión. En cambio, en el episodio de la sesión 5 no se logra el nivel de reflexión esperado, obteniéndose un nivel de generalización. Mencionamos dos posibles razones para que no se logre el nivel de reflexión, o bien el modelo de dependencia entre variables aún es emergente en los estudiantes y no ha llegado a nivel de la actividad formal, o bien no se desarrolló el proceso de reflexionar sobre la modelización por parte de los estudiantes. Estas dos explicaciones provenientes del MCM tienen un componente tanto curricular como de gestión: curricular en el sentido de que la resolución de la discrepancia entre el nivel esperado y el alcanzado puede abordarse desde la planificación, adaptando las actividades para que puedan permitir que emerja y se desarrolle un proceso de reflexión sobre la modelización y/o que el nivel de la actividad sea más formal; en cuanto al componente gestión del docente, este también es clave en la movilización de los procesos, y si bien Valentina genera intervenciones y preguntas para promover el proceso de reflexionar, quizás estas no fueron las adecuadas para un desarrollo de dicho procesos en los estudiantes.

\section{CONCLUSIONES}

En el MCM la competencia de modelización tiene un carácter distinto a las identificadas en otros trabajos. Algunos trabajos han asociado la competencia de modelización con las fases de modelización (Kaiser, 2007); en nuestro estudio, los ocho procesos que conforman la competencia modelización no tienen una correspondencia completa con las fases de modelización, ya que estos procesos pueden darse en cualquier fase. Por tanto, siguiendo a Maßß (2006), la competencia de modelización se desarrolla transversalmente a la secuencia que siguen las fases de modelización. Otros trabajos asocian las competencias a niveles de complejidad (Henning y Keune, 2007), pero en el MCM el nivel de complejidad se determina por medio de la articulación entre procesos, tareas y fases de modelización. La complejidad de una actividad no depende solo de su diseño, es fundamental su implementación en el aula de matemáticas, ya que se evidencian los procesos y las fases de modelización que se ponen en juego.

Los ocho procesos que conforman la competencia de modelización se han caracterizado cuando se introducen las funciones desde la interpretación de gráficas funcionales y no necesariamente emergen de la misma manera con una conceptualización diferente de las funciones. En la implementación de la unidad didáctica pudimos observar que uno de los procesos más frecuentes es interpretar el modelo, que a su vez es una tarea matemática que se presenta en varias actividades de la unidad didáctica, refle- 
jando así la importancia y el carácter dual que tiene la acción de interpretar. En cambio, si se introducen las funciones desde una notación $f(x)=m x+n$, las gráfica quedan supeditadas a la acción de construir desde la expresión algebraica, en que el proceso de construir la expresión del modelo se daría con mayor frecuencia que el de interpretar. Del mismo modo, para otros contenidos matemáticos algunos procesos pueden mantenerse pero otros se modificarán; los procesos que conforman la competencia de modelización no son transferibles a otros contenidos porque dependen de una estructura matemática.

Las fases de modelización han sido insertadas en la competencia de modelización utilizando la perspectiva de modelización emergente de Gravemeijer (2007), marco que ha sido un buen referente dado su carácter didáctico. Compartimos el hecho de que los modelos no cambian, sino que son los significados sobre el modelo los que cambian; ello se puede ver reflejado en el modelo de dependencia de variables que se utiliza en las diferentes etapas de la unidad didáctica. La clasificación modelo de/ modelo para se evidencia en el desarrollo de la unidad didáctica, dado que se observa un progreso continuo desde un predominio de un nivel de la actividad referencial hasta un predominio de un nivel general.

Algunos componentes del MCM pueden ser determinados a partir del diseño de la actividad y ser comprobados en el desarrollo de la actividad en el aula. En efecto, las tareas matemáticas son variables de la actividad, se definen en el diseño de la unidad didáctica y son desarrolladas en la implementación de las actividades en el aula. En cambio los procesos matemáticos son variables del sujeto y emergen en la interacción del aula entre estudiantes y profesor. En el MCM se ha modificado esta característica de los procesos; la competencia de modelización actúa como un instrumento que permite caracterizar los procesos matemáticos en la interacción del aula para que puedan ser considerados en el diseño de las actividades, lo que implica que estos pasen de ser una variable de sujeto a una variable de la actividad. Este cambio tiene como repercusión que los procesos que conforman una competencia puedan ser identificados en la planificación de una unidad didáctica. El tercer componente, las fases de modelización, es una variable de sujeto y se identifica solamente en la aplicación de la actividad en el aula y no en el diseño de la unidad didáctica. Estas características hacen que la competencia de modelización tenga una función didáctica puesto que la caracterización de sus componentes es una estructura útil tanto para la planificación de una secuencia didáctica, como para el desarrollo de la modelización en el aula.

Determinar el nivel de complejidad esperado en una actividad en función de una competencia es una contribución a la discusión sobre los niveles de complejidad. Los criterios que se describen en la literatura para clasificar una actividad en un nivel de complejidad u otro en ocasiones son ambiguos y difícilmente operativos. El informe PISA (OECD, 2012) ha avanzado tanto en esta discusión, estableciendo ciertos criterios para identificar la complejidad en la modelización relacionados con la existencia del modelo, como en la cantidad de restricciones para que el modelo satisfaga al problema. En nuestra propuesta se ha logrado que estos criterios sean operativos, proponiendo clasificar una actividad en función de la relación entre los componentes de la competencia. En consecuencia, consideramos que una contribución importante a la discusión sobre los niveles de complejidad es definir criterios para determinar el nivel de complejidad esperado en una actividad en función de una competencia. Si bien estos criterios de complejidad para la competencia de modelización se desprende de un estudio de casos y no se puede generalizar, en otros estudios (Solar et al., 2012) se han obtenido criterios bajo el mismo método de análisis para la competencia de argumentación, evidenciando que el MCM poder ser aplicado para distintas competencias y casos.

Destacamos, como elemento fundamental en el modelo de competencia matemática (MCM), los procesos matemáticos, ya que son estos los que conforman una competencia matemática. Si bien las organizaciones matemáticas también son parte del modelo, éstas representan a los contenidos que han estado explícitamente presentes en los currículos. En cambio, los procesos han estado históricamente 
ausentes en la organización del diseño curricular. El MCM equilibra la importancia entre las tareas matemáticas y los procesos: las tareas se desarrollan a corto plazo y cambian en los diferentes niveles del currículo, en cambio los procesos se repiten y se desarrollan a largo plazo en el currículo de matemáticas.

\section{REFERENCIAS BIBLIOGRÁFICAS}

Abrantes, P. (2001). Mathematical competence for all: Options, implications and obstacles. Educational Studies in Mathematics, 47, pp. 125-143. http://dx.doi.org/10.1023/A:1014589220323

Blomнøj, M. y HøjgaARd, T. (2003). Developing mathematical modelling competence: Conceptual clarification and educational planning. Teaching Mathematics and its Applications, 22(3), pp. 123139.

http://dx.doi.org/10.1093/teamat/22.3.123

Blum, W.; Galbraith, P. L.; Henn, H.-W. y Niss, M. (2007). Modelling and applications in mathematics education. The 14th ICMI Study. New York: Springer.

Brousseau, G. (1997). Theory of Didactical Situations in Mathematics. The Netherlands: Kluwer Academic Publishers.

Chevallard, Y. (1999). L'analyse des practiques enseignantes en théorie anthropologique du didactique. Recherches en Didactique des Mathématiques, 19(2), pp. 221-266.

De Lange, J. (1987). Mathematics: Insight and meaning. Utrecht: OW y OC.

De Lange, J. (1995). Assessment: No change without problems. En T. A. Romberg (ed.). Reform in school mathematics and authentic assessment. New York: SUNY Press, pp. 87-172.

Espinoza, L.; Barbé, J.; Cerda, F.; Waisman, F.; Ruiz, R. y Gálvez, G. (2006). Analizando y construyendo gráficas. Unidades didácticas LEM. Santiago: Mineduc.

Espinoza, L.; Barbé, J.; Mitrovich, D.; Solar, H.; Rojas, D. y Matus, C. (2008). Análisis de las competencias matemáticas en primer ciclo. Caracterización de los niveles de complejidad de las tareas matemáticas. FONIDE N.o: DED0760. Santiago: Mineduc.

Gravemeijer, K. (2007). Emergent modelling as a precursor to mathematical modelling. En W. Blum, P. L. Galbraith, H.-W. Henn y M. Niss (eds.). Modelling and applications in mathematics education. The 14th ICMI Study. New York: Springer, pp. 137-144.

Gravemeijer, K. y Doorman, M. (1999). Context problems in realistic mathematics education: A calculus course as an example. Educational Studies in Mathematics, 39, pp. 111-129. http://dx.doi.org/10.1023/A:1003749919816

Henning, H. y Keune, G. (2007). Levels of modeling competence. En W. Blum, P. L. Galbraith, H.-W. Henn y M. Niss (eds.). Modelling and applications in mathematics education. The 14th ICMI Study. New York: Springer, pp. 225-232.

Houston, H. (2007). Assessing the "phases» of mathematical modeling. En W. Blum, P. L. Galbraith, H.-W. Henn y M. Niss (eds.). Modelling and applications in mathematics education. The 14th ICMI Study. New York: Springer, pp. 249-256.

KaIser, G. (2007). Modelling and modelling competencies in school. En C. Haines, P. Galbraith, W. Blum y S. Khan (eds.). Mathematical Modelling. The 12th ICTMA Study. Education, Engineering and Economics. Chichester: Horwood, pp. 110-119.

Leinhardt, G.; Zaslavsky, O. y Stein, M. K. (1990). Functions, graphs, and graphing: Task, learning, and teaching. Review of Educational Research, 60, pp. 1-64.

http://dx.doi.org/10.3102/00346543060001001 
MAAß, K. (2006). What are modelling competencies? Zentralblatt für Didaktik der Mathematik, 38(2), pp. 113-142.

Ministerio de Educación, Cultura y Deporte (2013). Marcos y pruebas de evaluación de PISA 2012: Matemáticas Lectura y Ciencias. Madrid: Secretaría general técnica.

National Council of Teachers of Mathematics (nctm). (2003). Principios y Estándares para la Educación Matemática. Sevilla: Sociedad Andaluza de Educación Matemática Thales.

Niss, M. (2002) (coord). Mathematical competencies and the learning of mathematics: The danish kom project. Roskilde: Roskilde University.

Niss, M. y HøjgaARD, T. (2011). Competencies and Mathematical Learning: Ideas and inspiration for the development of mathematics teaching and learning in Denmark. Roskilde: Roskilde University.

OECD (2003). The PISA 2003 Assessment Framework Mathematics, Reading, Science and Problem Solving Knowledge and Skills. Paris: OECD.

Puig, L. (2008). Sentido y elaboración del componente de competencia de los modelos teóricos locales en la investigación de la enseñanza y aprendizaje de contenidos matemáticos específicos. PNA, 2(3), pp. 87-107.

Rico, L. (2007). La competencia matemática en PISA. PNA, 1(2), pp. 47-66.

Solar, H.; Azcárate, C. y Deulofeu, J. (2009). Competencia de modelización en la interpretación de gráficas funcionales. En M. J. González; M. T. González y J. Murillo (eds.). Investigación en Educación Matemática XIII. Santander: SEIEM, pp. 499-510.

Solar, H.; Azcárate, C. y Deulofeu, J. (2012). Competencia de argumentación en la interpretación de gráficas funcionales. Enseñanza de las Ciencias, 30 (3), pp. 133-154.

Solar, H.; Rojas, F.; Ortiz, A. y Ulloa, R. (2012). Reflexión docente y competencias matemáticas: un modelo de trabajo con docentes. RECHIEM: Revista Chilena de Educación Matemática, 6 (1), pp. 257-267. 


\title{
Modeling competence in functional graph interpretation
}

\author{
Horacio Solar \\ Departamento de Didáctica, Pontificia Universidad Católica de Chile. \\ hsolar@uc.cl \\ Jordi Deulofeu \\ Universitat Autònoma de Barcelona \\ jordi.deulofeu@uab.cat \\ Carmen Azcárate \\ Departament de Didàctica de les Matemàtiques i les Ciències Experimentals, Universitat Autònoma de Barcelona. \\ carmen.azcarate@uab.cat
}

In this paper we interpret the modeling in graph interpretation as a mathematical competence. We have generated a mathematical competence model that is useful to plan a teaching sequence, and also to analyze its development in the mathematics class. This model is elaborated from topic of graph interpretation, as we start assuming that the mathematical competencies aren't independent of the mathematical contents. Also, as competencies are developed through the mathematical processes, we intend to know the relation between processes and contents, and also whether the processes change depending on the school level.

The Mathematical Competence Model (MCM), based on processes, tasks and levels of complexity, is tested in the study of modeling competence, in a mathematical class in which a teaching unit about functional graph interpretation is applied. Our central research question is: How are tasks, processes and complexity related in the development of modeling competence?

The MCM is presented with the following features:

- Mathematical competence: the mathematical competencies are processes that articulate and organize the curriculum at diverse levels: modeling, representation, problem solving, argumentation, etc.

- Mathematical organizations: we propose the mathematical contents to be structured in terms of mathematical organizations and composed of four categories: mathematical tasks, techniques, technology and theory.

- Levels of complexity: the competence progress is determined in terms of activity complexity, which depends on the tasks and also on the processes that shape them.

The MCM is tested in an experimental study. We studied the case of Valentina, an $8^{\text {th }}$ grade teacher (14-15 years old) who applied a teaching unit called «Analyzing and constructing graphs».

The application of the teaching unit was followed with a strategy of non participating observation during five lessons. Using video recordings, records were considered focusing on the interaction between teacher and students. Finally, the five classes recorded were transcribed.

Episodes that show a greater richness in the interaction between teacher and students were selected from each lesson. The unit of analysis corresponded to each of the actions of the episode, both for Valentina and the students. Each action is characterized by a term indicating the process associated with competence. The strategy followed an approach of constant comparison between the processes that emerged from the analyzed episodes, concluding with the characterization of a process list for modeling competence.

Eight processes conform the modeling competence: Characterization of the model, validation of the model characteristics, interpretation of the model, implementation of an expression of the model, identification of the model properties, application of the model, validation of the model, and reflection about the modeling.

In the maps of processes, it is evident that the more frequent ones are to characterize the model and to interpret the model. The validation processes generally emerge from the teacher. The most frequent is to validate the characteristics of the model and the process to validate the model hardly ever occurs; this last process is unlikely to emerge because it requires high learning levels of formalization and abstraction.

The rest of the processes emerge in an occasional way, due to the fact that they act in punctual moments. We suppose that the process to identify the properties of the model frequently occur because the properties are generally discussed in reference to the real model or to the context, without a special interest to do it in the mathematical model. In addition, the process to implement the model appears not so frequently because generally the use of the model occurs in actions linked to other processes like to interpret or to construct the expression of the model, but it has been difficult to find actions focused on applying the model already known.

The results have shown that there is a base structure: the levels of complexity identify the cognitive level of a mathematical task regarding the process.

From the results of the modeling competence, we set out that the development of this competence depends mainly on the planning, in the sense that based on the kind of activity and on the teaching sequence, it is possible to determine processes and levels of complexity. 